\title{
Hits and false positives in face matching: A familiarity-based dissociation
}

\author{
Ahmed M. Megreya \\ Menoufia University, Shebin El Kom, Menoufia, Egypt \\ AND \\ A. Mike Burton \\ University of Glasgow, Glasgow, Scotland
}

\begin{abstract}
In recognition memory for unfamiliar faces, performance for target-present items (hits) does not correlate with performance for target-absent items (false positives), a result which runs counter to the more usual mirror effect. In this paper we examine subjects' performance on face matching, and demonstrate no relationship between performance on matching items and performance on nonmatching items. This absence of a mirror effect occurs for multidistractor, 1-in-10 matching tasks (Experiment 1) and for simple paired-item tasks (Experiment 2). In Experiment 3 we demonstrate that matching familiar faces produces a strong mirror effect. However, inverting the familiar faces causes the association to disappear once more (Experiment 4). We argue that familiar and unfamiliar faces are represented in qualitatively different ways.
\end{abstract}

The mirror effect is a well-established characteristic of recognition memory (see Glanzer \& Adams, 1985; Glanzer, Adams, Iverson, \& Kim, 1993, for reviews). It can be summarized as follows: "If there are two classes of stimuli, and one is more accurately recognized than the other, then the superior class is both more accurately recognized as old when old and also more accurately recognized as new when new" (Glanzer \& Adams, 1990, p. 5). This effect is very robust and has been demonstrated across a wide variety of stimulus categories. It has attracted a great deal of theoretical attention, and a number of distinct theories have been proposed to account for it (e.g., Glanzer et al., 1993; Joordens \& Hockley, 2000; Malmberg \& Nelson, 2003; McClelland \& Chappell, 1998; Reder et al., 2000; Shiffrin \& Steyvers, 1997; Sikström, 2004).

Despite this well-established literature, there is one class of stimulus which appears not to demonstrate a mirror effect. In recognition memory experiments for unfamiliar faces, researchers have generally found no association between hits, identifying old faces as being old, and false positives (FPs), identifying new faces as being old (Bruce, Burton, \& Dench, 1994; Hancock, Burton, \& Bruce, 1996; Lewis \& Johnston, 1997; Vokey \& Read, 1992). This is perhaps a counterintuitive finding: One might expect that a "distinctive" face would be easy to recognize at test (if indeed it had been seen before) and equally easy to reject (if it had not been seen), leading to a reliable negative correlation between hits and FPs. However, this is not the case.
Correlational research with faces is not conducted in the same manner as a typical mirror-effect experiment. However, even when face memory items are manipulated into two categories - easy and hard items - as with mirror-effect experiments (e.g., by using same or otherrace faces; O'Toole, Deffenbacher, Valentin, \& Abdi, 1994; or by using faces with and without sunglasses, Hockley, Hemsworth, \& Consoli, 1999), there is evidence that the normal association between hits and FPs breaks down.

Vokey and Read (1992) investigated the relationship between distinctiveness and recognition memory for faces. They first asked subjects to rate faces for a number of dimensions: typicality, familiarity (i.e., how similar the faces were to those of people known by the subjects), memorability (i.e., how easy to remember subjects thought the face would be), attractiveness, and likability. These ratings were subject to principal components analysis (PCA), and two orthogonal dimensions were extracted. The authors labeled these general familiarity and memorability. The typicality ratings loaded equally on these two dimensions, leading the authors to argue that "distinctiveness" is not a unitary concept but includes both these factors.

In subsequent experiments, Vokey and Read (1992) examined the relationship between these two components and face recognition memory. At learning, subjects were asked to rate the likability of a set of unfamiliar faces. At test, they were shown the same or different images of these targets, along with a new set of distractors. Interestingly, the two components affected recognition in opposite

A. M. Burton, mike@psy.gla.ac.uk 


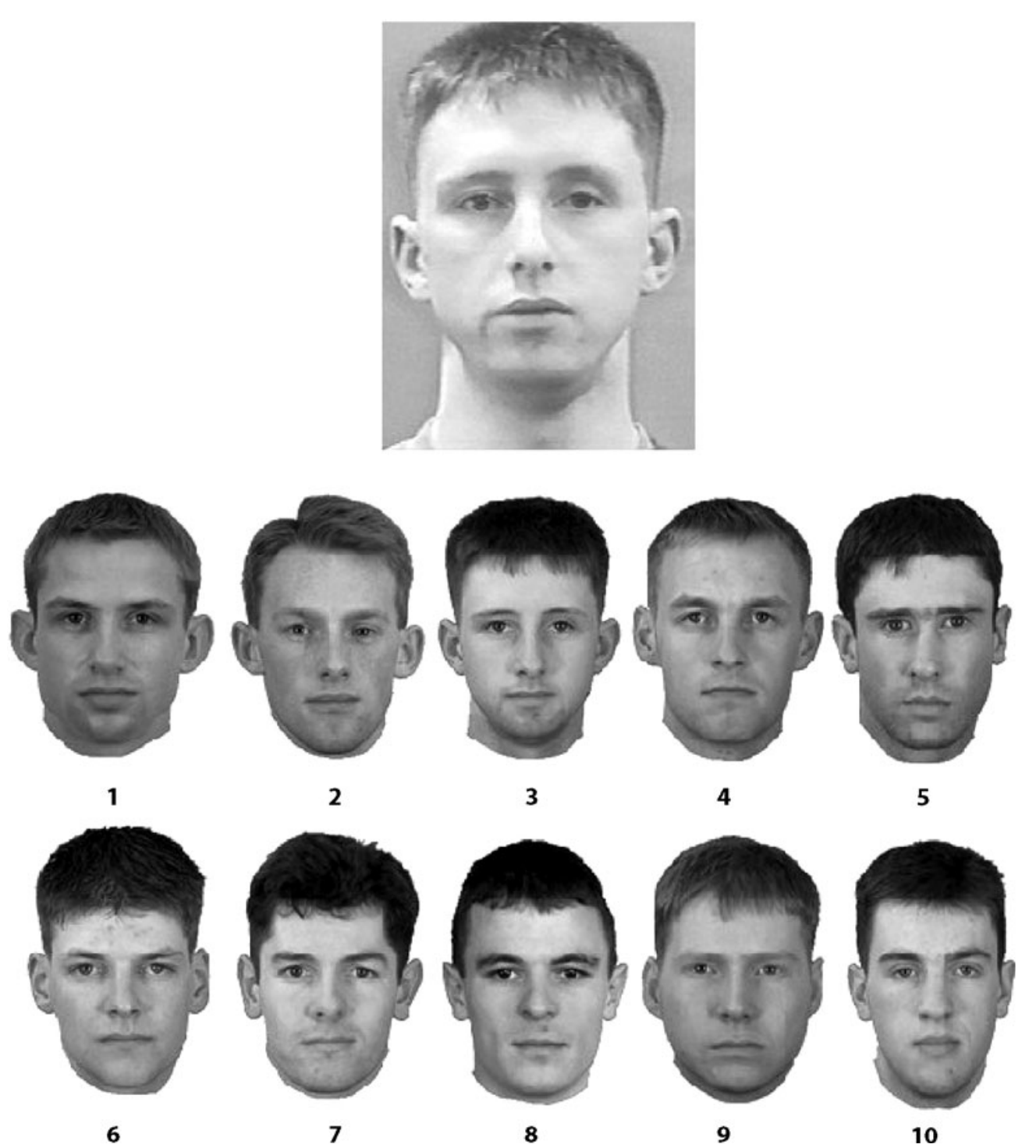

Figure 1A. The person shown at the top may or may not be one of the ten below. The subject's task is to decide whether the target is present and, if so, which he is. From "Verification of Face Identities From Images Captured on Video" by Bruce et al., 1999, Journal of Experimental Psychology: Applied, 5, p. 344. Copyright 1999 by the American Psychological Association. Reprinted with permission.

directions. Recognition was increased by increasing memorability, but decreased by increasing general familiarity. More specifically, general familiarity had a significant positive effect on FPs but had no effect on hits. On the other hand, memorability had a significant negative effect on FPs but had no effect on hits. In other words, "generally familiar" faces elicit hits whether or not they have been seen, whereas "memorable" faces reduce FPs. Although the details of this proposal have been challenged (Deffenbacher, Johanson, Vetter, \& O'Toole, 2000), explanations of performance in recognition memory for faces continue to rely on dual-process accounts in which at least one component relies on memorability of the stimuli.

In this article, we examine the relationship between hits and FPs in face matching. The intention is to examine whether faces are subject to the normal mirror effect when stimuli are present simultaneously. Intuitively, one might predict that a face that is easy to match - that is, the two images seem to be of the same person-would also be easy to reject when the two images are not of the same person. In a matching task with no time constraint, any effects of memorability may be minimized; theories used to explain no association in face memory might not, therefore, be applicable to matching. Nevertheless, our previous face-matching experiments have suggested an absence of correlation between hits and FPs (Bruce et al., 1999; Megreya \& Burton, 2006b).

In fact, unfamiliar face matching is a more difficult task than is commonly understood. Bruce et al. (1999) showed subjects arrays of the sort presented in Figure 1. A target unfamiliar face was presented above a lineup consisting of 10 faces, one of which might be the target. The targets 
and the 10-face lineup were seen simultaneously, under no time pressure, and the target and lineup images were taken in good lighting conditions, showing a full-face view with neutral expression, and taken on the same day, eliminating any minor differences in appearance such as hairstyle, weight, or age. However, crucially, they were taken with different cameras, so superficial image characteristics differ. Bruce et al. (1999) found that matching performance was, surprisingly, very poor. When the target was present in the lineup, subjects picked the correct match on only about $70 \%$ of occasions. When the target was absent, they still chose a face $30 \%$ of the time.

These results have been replicated on a number of occasions, and similarly poor performance is obtained, even when the task is reduced to a 10AFC with no target-absent arrays (Burton, Miller, Bruce, Hancock, \& Henderson, 2001), or when simple two-item match/mismatch trials are used (Bruce, Henderson, Newman, \& Burton, 2001; Henderson, Bruce, \& Burton, 2001; Megreya \& Burton, 2006b). Furthermore, in a "live" matching task, Kemp, Towell, and Pike (1997) found that shop workers were very poor in matching I.D. card photographs to their bearers.

This difficulty of unfamiliar face matching has been taken to suggest that processing of unfamiliar faces relies on image matching rather than on a more sophisticated face matching strategy available for familiar faces (Hancock, Bruce, \& Burton, 2000). Furthermore, Megreya and Burton (2006b) show that there are large and stable individual differences in people's performance on unfamiliar face matching, and that this does not correlate with their performance on upright familiar face matching. Rather, it correlates positively and strongly with matching inverted faces, whether or not they are familiar or unfamiliar; this supports the conclusion of Hancock et al. (2000) that unfamiliar face processing is image based.

In the first experiment reported here, we used the 1-in-10 face matching task to provide a test for the absence of a mirror effect in face matching. Previous studies suggesting this result (Bruce et al., 2001; Megreya \& Burton, 2006b) have not been designed to allow analysis by face, whereas the memory studies of Vokey and Read (1992) did allow this analysis. Therefore, in the following experiment, each subject was presented with every target face in both target-present and target-absent arrays. In this way, the relationship between hits and FPs elicited to the same faces could be examined by analyzing data collected by both subjects - are the subjects with high hits also those with low FPs? - and items - are those faces which generally have high hit levels also those with low FP levels? In the second experiment, we examined this relationship using a simple paired-item matching task. Finally, we report two further experiments in which we examined this relationship using upright and inverted familiarized face matching tasks by both by-subject and by-item analysis.

\section{EXPERIMENT 1}

In this first experiment, subjects were presented with the normal 1-in-10 face matching task, in which the tar- gets were present only in half the arrays. Immediately after completing this task, subjects were presented with an identical task, except that targets that had been seen in target-present trials in the first task were now seen in target-absent trials. Similarly, targets seen in target-absent trials in the first task were now seen in target-present trials. Using this technique, we can examine the relationship between hits in one task and FPs in the other, all elicited by the same faces.

\section{Method}

Subjects. Thirty students from the University of Glasgow, 18 women and 12 men, age range 17-21, participated in the experiment for payment or course credits. All had normal or corrected-tonormal vision.

Stimuli and Procedure. One hundred sixty face-matching arrays of those produced by Bruce et al. (1999) were used as stimuli. Each array consisted of a target face and 10-face lineup. The targets were images captured with a high quality video camera, whereas the lineup images were high quality photographs captured by a studio camera. The target and lineup images were taken on the same day; each image showed a very similar full-face pose of a young, cleanshaven Caucasian man. All images were presented in gray scale, and the size of each was approximately $5 \times 7 \mathrm{~cm}$. The images were taken from the U.K. Home Office Police Information Technology Organization (PITO) database. Full details of array construction are given in Bruce (1999). In brief, the faces came from a graduating class of 120 police officers. Distractors were chosen to be the most similar items to the target, on the basis of a prior similarity ratings study, so distractors were relatively similar to targets (see Figure 1), to the extent that similar people may be found in a class pool of 120 young men.

Two sets of stimuli were constructed from these arrays, so that each set consisted of 50\% target-present arrays and 50\% target-absent arrays. Each subject was presented with one set, which included 80 trials: 40 target-present and 40 target-absent arrays. Immediately after completing this set, they were presented with the other set of stimuli, so that the target faces which had appeared in target-present trials in the first set were now presented in target-absent arrays, and vice versa. Thus, over the course of the experiment each subject saw every face in both target-present and target-absent trials. The sets were blocked and counterbalanced in the order of presentation.

The experiment was run on a G3 Macintosh computer using Superlab Pro software and lasted approximately $50 \mathrm{~min}$. Each array was presented on the screen until subjects responded, and there was a 1-sec interstimulus interval (ISI). The order of stimuli within each set was independently randomized for each subject. The subjects had to match the target face for identity with the 10 candidates presented underneath; 11 labeled keys on the standard computer keyboard were used to record subjects' responses. Subjects were informed that targets would be present on half the occasions and that there was no time limit for making a decision. They were encouraged to perform as accurately as possible.

\section{Results}

The face-matching data has been broken down into three target-present measures: hits (i.e., identifying the correct match), misses (i.e., the false negative response that the target is absent), and misidentifications (i.e., identifying a wrong face as a match in the presence of the correct match). For target-absent trials, FPs (i.e., identifying a wrong face as a match in the absence of the correct match) were calculated. Table 1 shows the differences between subjects' performance on the two sets of face matching stimuli; Table 2 
Table 1

Matching Performance for the Two Sets of Stimuli in Experiment 1 and a $t$-Test Comparison Between the Two Sets

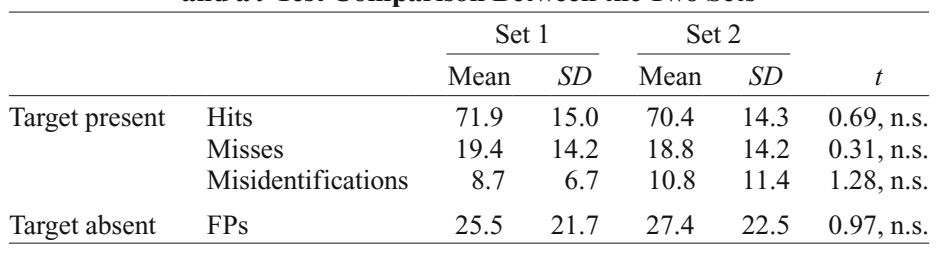

Note $-N=30$. FPs, false positives.

shows Pearson's correlation coefficients between subjects' performance on these sets.

Within each set of stimuli, there was no correlation between hits and FPs $[r \mathrm{~s}(28)=-.11$ and $-.26, p>.05$, for Sets 1 and 2, respectively]. In contrast, there were high positive associations between FPs and misidentifications $[r \mathrm{~s}(28)=.87$ and $.86, p<.01$, for Sets 1 and 2 , respectively].

Across the two sets, by-subject analysis showed no correlation between hits in one set and FPs in the other, and high positive associations between FPs in one set and misidentifications in the other (see Table 2). Similarly, the by-item analyses showed no relationship between hits in Set 1 and FPs in Set $2[r(38)=.03, p>.05]$, nor between hits in Set 2 and FPs in Set $1[r(38)=.01, p>.05]$. In addition, there were high positive associations between misidentifications in Set 1 and FPs in Set $2[r(38)=.62$, $p<.01]$ and between FPs in Set 1 and misidentifications in Set $2[r(38)=.54, p<.01]$.

\section{Discussion}

Overall levels of performance on the matching task are roughly $70 \%$, replicating other research with these stimuli (Bruce et al., 1999; Megreya \& Burton, 2006a, 2006b). Subjects' performance on the two sets of face matching stimuli was very similar. Furthermore, there were strong positive associations between the two sets.

Within each stimulus set, there were strong positive associations between FPs and misidentifications using the by-subject analysis. This finding suggests that people who misidentified the targets when they were present in the lineups were highly likely to choose an incorrect face in the target-absent lineups. Further, it suggests that faces that elicited misidentification in target-present trials were highly likely to elicit FPs in target-absent trials. The byitem analysis across stimulus sets provided strong support for this suggestion, as there were strong positive associa- tions between FPs in one set and misidentifications in the other set, which consisted of the same target faces. This is consistent with a study by Wells (1993), who found that when the target was removed from the lineup without replacement, most eyewitnesses picked a foil which was the second-best choice. Clark and Davey (2005) replicated this so-called target-to-foils shift, which was equal in size for simultaneous and sequential lineups.

Of most interest here is the fact that there was no reliable association between hits and FPs in matching the faces within both sets. This lack of negative correlation between hits and FPs is well established in the face-memory literature (Bruce et al., 1994; Hancock et al., 1996; Lewis \& Johnston, 1997; Vokey \& Read, 1992). However, the present experiment confirms suggestions in our previous experiments (Bruce et al., 1999; Megreya \& Burton, 2006b) that the pattern is the same for a matching task.

In general, the similar level of difficulty in the two stimulus sets indicates that the absence of a mirror effect across these sets is not a surprise, as the effect does require that one set should be harder in recognition than the other (see Glanzer \& Adams, 1985; Glanzer et al., 1993, for reviews). However, the situation here is rather different. The target faces were the same in the two sets, but the results still showed no correlation between hits and FPs. Therefore, faces that were easy to match in target-present arrays did not retain this advantage in target-absent trials. For example, $67 \%$ of subjects made FPs for the array shown in Figure 1B, but when the same target was present in the lineup, about $80 \%$ of the same subjects picked up the correct match.

This experiment provides a first indication that the absence of a mirror effect for faces is a more complex problem than previously understood. However, the particular matching task we have used - the 1-in-10 lineup task-perhaps has more in common with a standard recognition memory procedure than with a simple two-item match. The large number of distractors in this matching

Table 2

Pearson's Correlation Coefficients Between Subjects' Performance on the Two Face-Matching Sets in Experiment 1

\begin{tabular}{llcccr}
\hline \multicolumn{6}{c}{ on the Two Face-Matching Sets in Experiment 1 } \\
\hline \multirow{2}{*}{ Target present } & Hits & $.64^{* *}$ & $-.56^{* *}$ & -.23 & -.19 \\
& Misses & $-.59^{* *}$ & $.74^{* *}$ & -.23 & $-.41^{*}$ \\
& Misidentifications & -.05 & -.22 & $.58^{* *}$ & $.74^{* *}$ \\
Target absent & FPs & -.07 & -.28 & $.75^{* *}$ & $.88^{* *}$ \\
\hline Note $-N=30$. FPs, false positives. & ${ }^{*} p<.05$. & ${ }^{* *} p<.01$. & &
\end{tabular}




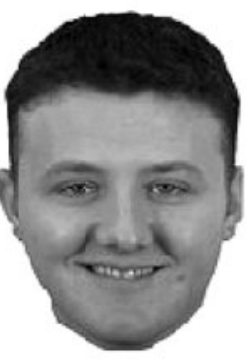

1

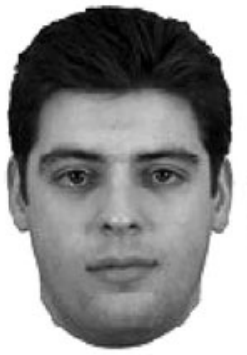

6

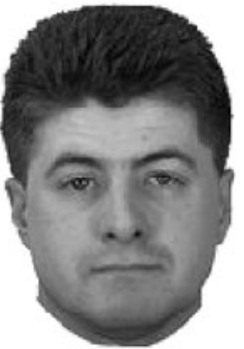

2

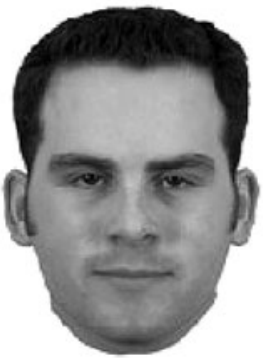

7

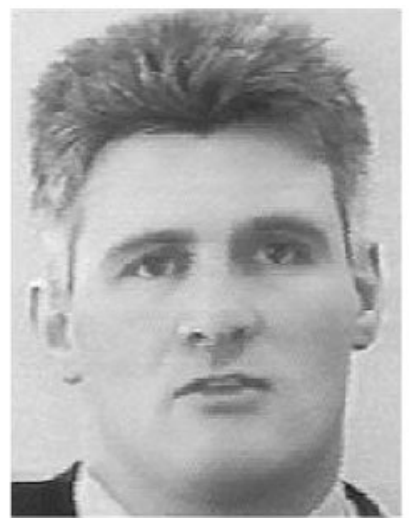

Figure 1B. The person shown at the top may or may not be one of the ten below. The subject's task is to decide whether the target is present and, if so, which he is. From "Verification of Face Identities From Images Captured on Video" by Bruce et al., 1999, Journal of Experimental Psychology: Applied, 5, p. 344. Copyright 1999 by the American Psychological Association. Reprinted with permission.

task may in some way link matching and memory; indeed, we have previously found modest positive correlations between matching and memory tasks with faces (Megreya $\&$ Burton, 2006b). This may produce the similar pattern of dissociation between hits and FPs in unfamiliar face recognition. In the following experiment, we examine a reduced matching task.

\section{EXPERIMENT 2}

In Experiment 1 we did not find an association between hits and FPs using a 1-in-10 face-matching task. In the present experiment, we reduced the heavy demands of the task by using a simple two-item matching procedure. This reduces the inherent immediate memory load on the subjects, and substantially reduces the possible influence of multiple distractors.

\section{Method}

Subjects. Forty paid subjects, 25 women and 15 men, age range 18-27, participated in this experiment. All subjects were students at the University of Glasgow. None had taken part in the previous experiments. All had normal or corrected-to-normal vision.

Stimuli and Procedure. The stimuli were 84 pairs of unfamiliar faces, half of which matched, and half of which did not. Examples are given in Figure 2. All images were of the same size (approximately $5 \times 7 \mathrm{~cm}$ ), and were cropped by graphic software to remove the background and clothes.

The experiment was run on a G3 Macintosh computer using Superlab Pro software. Each pair of faces was presented on the screen until subjects responded, and there was a 1-sec ISI. The order of the stimuli was randomized independently for each subject. The subjects' task was to decide whether the two images were of the same person or of two different people by pressing one of two labeled keys on the keyboard. Each subject completed 84 trials: 42 match and 42 mismatch. Presentation of targets was counterbalanced across the experiment; across subjects, therefore, each identity appeared 

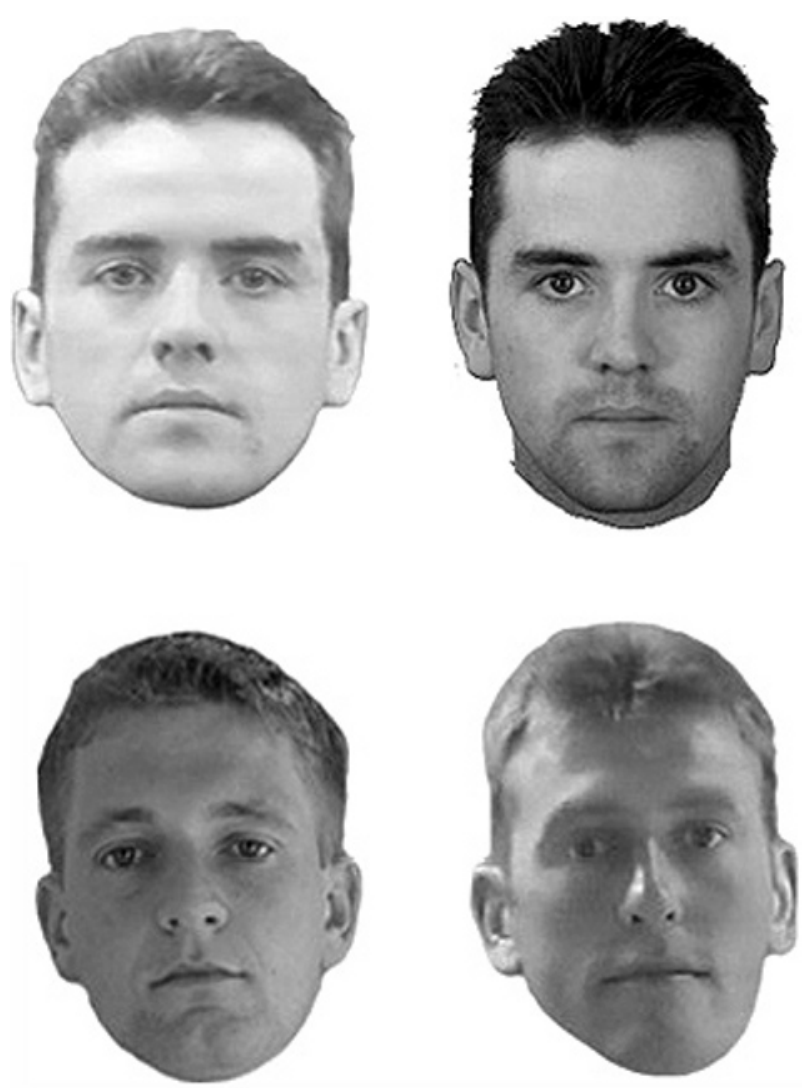

Figure 2. Examples of match and mismatch face pairs used in Experiment 2.

equally often in match and mismatch trials. Subjects were encouraged to perform as accurately as possible.

\section{Results}

Mean performance was $78 \%$ accurate on match trials $(S D=17)$ and $84 \%$ accurate on mismatch trials $(S D=$ 12). There was no correlation between hits and FPs, either by subjects $[r(38)=.11, p>.05]$ or by faces $[r(82)=$ $.10, p>.05]$.

\section{Discussion}

Once again, performance on this task was poor, confirming previous research (Bruce et al., 1999, 2001; Henderson et al., 2001). This overall performance demonstrates that the difficulty of the 1-in-10 lineup task does not lie wholly in the large number of distractors, a finding also demonstrated by Megreya and Burton (2006a); rather, we have argued, it lies in problems of encoding unfamiliar faces. These problems are emphasized when the surface characteristics of images are different - that is, when photographs are taken with different cameras.

Of greatest interest for the present article is the lack of correlation between performance in match and mismatch trials. This finding was observed in both by-subject and by-item analyses, adding support to our proposal that the ability to match a face is unrelated to the ability to reject that face. Furthermore, the fact that this pattern persists even when the number of distractor items is radically reduced suggests that the pattern generalizes across different tasks with faces, rather than being due to task difficulty.

This is an intriguing finding. If we consider familiar faces, it seems intuitively reasonable to suggest that performance on present and absent trials would be equivalent. For example, it should be relatively easy to match two images of Tony Blair, or to say whether one of the faces is not Tony Blair. In fact, it is very difficult to make the comparison between familiar and unfamiliar face matching, precisely because familiar face matching tasks almost always give rise to ceiling levels of performance (Burton, Wilson, Cowan, \& Bruce, 1999; Liu, Seetzen, Burton, \& Chaudhuri, 2003; Megreya \& Burton, 2006b). In the next experiment, we solve this problem by using experimentally familiarized faces and examining matching performance on them.

\section{EXPERIMENT 3A}

This experiment examined the effect of familiarity on the relationship between hits and FPs in matching faces. Using rather different tasks, Experiments 1 and 2 have consistently shown that hits and FPs do not correlate with each other in matching unfamiliar faces. The present experiment aims to examine the extent to which this finding will generalize to familiar faces. To do so, we return to the 1-in-10 lineup task.

The target images, at the top of arrays illustrated in Figure 1, are stills from a high quality video. The original source videos from this database comprise 30-sec clips of subjects moving their heads left to right, up and down, and nonrigidly through talking (though no sound is present). This gives us the opportunity to familiarize subjects with the target people before showing the matching arrays. This is a manipulation which, despite the relatively brief exposure, has been shown to improve subjects' matching ability significantly (Bruce et al., 2001; Megreya \& Burton, $2006 \mathrm{~b}$ ), but not to the ceiling levels which would be present for highly familiar faces such as those of celebrities. The advantage of this procedure is that it allows us to compare familiar and unfamiliar faces on the same task, and (through counterbalancing of stimuli) across the same faces.

This study consists of two subexperiments. Experiment $3 \mathrm{~A}$ examined the relationship between hits and FPS in a by-subject analysis, whereas Experiment 3B examined this relationship by items.

\section{Method}

Subjects. Thirty University of Glasgow students, 20 women and 10 men, age range $17-25$, participated in this experiment. All were new to the task, and they were given either a small payment or course credits for participation. All had normal or corrected-tonormal vision.

Stimuli and Procedure. The arrays produced by Bruce et al. (1999) were split into two sets. One was used for the unfamiliar face-matching task and the other for familiarized face-matching. The two sets of faces were counterbalanced across the experiment 
Table 3

Mean Performance (\%) on Matching Unfamiliar and

Familiarized Faces (By-Subject Analysis) in Experiment 3A

\begin{tabular}{|c|c|c|c|c|c|c|c|c|c|c|}
\hline & \multicolumn{6}{|c|}{ Target-Present Trials } & \multicolumn{4}{|c|}{ Target-Absent Trials } \\
\hline & \multicolumn{2}{|c|}{ Hits } & \multicolumn{2}{|c|}{ Misses } & \multicolumn{2}{|c|}{ Misidentifications } & \multicolumn{2}{|c|}{ Correct Reject } & \multicolumn{2}{|c|}{ FPs } \\
\hline & $M$ & $S D$ & $M$ & $S D$ & $M$ & $S D$ & $M$ & $S D$ & $M$ & $S D$ \\
\hline Unfamiliar & 76 & 17 & 16 & 15 & 8 & 7 & 72 & 16 & 28 & 16 \\
\hline Familiarized & 87 & 9 & 9 & 7 & 4 & 5 & 88 & 10 & 12 & 10 \\
\hline
\end{tabular}

Note-FPs, false positives.

so that each face was seen equally often as unfamiliar or familiarized. Subjects were tested individually in a session of approximately $1 \mathrm{~h}$. The unfamiliar face-matching task was always performed first, followed by the familiarization phase, followed by the familiarized face-matching task. For each task, subjects completed 40 trials: 20 target-present and 20 target-absent trials.

For the familiarization procedure, each subject was shown 40 clips. In each clip a target was seen rotating gradually from $0^{\circ}$ (frontal view) to $90^{\circ}$ (a profile view), and from $270^{\circ}$ (the opposite profile) to $360^{\circ}$ (frontal again). Then each target looked up, looked down, and smiled toward the camera. The duration of each clip was approximately $30 \mathrm{sec}$, and they were played continually, with a 1-sec gap between videos. Subjects were instructed that they should study each face very carefully, and that they would be asked to recognize them in a later recognition test.

\section{Results}

Table 3 shows mean matching performance for unfamiliar and familiarized faces. There were significant differences in hits $[t(29)=3.78, p<.01]$ and FPs $[t(29)=4.93, p<.01]$ between matching familiarized and unfamiliar faces. There was no reliable correlation between hits and FPs for unfamiliar faces $[r(28)=.09, p>.05]$, but a large and significant correlation for familiar faces $[r(28)=-.71, p<.01]$.

\section{Discussion}

First we note that the familiarization process was successful. Overall performance rose in familiarized faces, by comparison to unfamiliar faces, which displayed levels of performance similar to those observed in Experiment 1. Of most interest, though, is the fact that familiarized faces show a very strong mirror effect. Unlike our previous experiments, and unlike the literature on recognition memory for unfamiliar faces, the familiar faces show a straightforward association between target-present and target-absent performance. Prior to familiarization, there was a close to zero correlation between hits and FPs, whereas this simple procedure of showing $30 \mathrm{sec}$ of video of targets produced a very strong association.

In Experiment 3A, faces were counterbalanced between the two familiarity conditions; this does not allow the relationship between hits and FPs by faces to be examined. With only 30 subjects in total, by-face correlations would be based on an $N$ of 15, providing little power. However, since the pattern shown above is novel, it is important to establish whether it exists in a by-face analysis. For this reason, in Experiment 3B we added more data to existing data from Experiments 1 and $3 \mathrm{~A}$ in order to give enough subjects to warrant a by-items correlational analysis.

\section{EXPERIMENT 3B}

\section{Method}

Subjects. Sixty-five new subjects, 41 women and 24 men, age range 17-25, participated in this experiment. All were students at the University of Glasgow. All subjects had normal or corrected-tonormal vision.

Stimuli and Procedure. We took one of the two sets of faces used in Experiment $3 \mathrm{~A}$ and ran a further 25 subjects on the same procedure as that used in Experiment 3A. Adding this to the existing 15 subjects run on this set (Experiment $3 \mathrm{~A}$ ) provided a total of 40 subjects' data for the particular faces familiarized in this set. We then compared matching performance on this set when familiarized, with matching performance on the same set when not familiarized. Therefore, we tested a further 40 subjects (who had not participated in any of the experiments presented above) using this same set of faces, but without familiarization.

\section{Results}

Table 4 shows mean matching performance for unfamiliar and familiarized faces. There was no reliable correlation between hits and FPs when the faces were unfamiliar $[r(38)=-.21, p>.05]$, but a large and significant correlation when they were familiarized $[r(38)=-.60$, $p<.01]$.

\section{Discussion}

The results are very similar to the by-subjects analyses in Experiment 3A. As expected, performance is better on familiarized than on unfamiliar faces (even when these are the same faces, tested across the experiment). More interestingly, there is a very large association between performance in target-present and target-absent trials, but only for the familiar faces. This lends further support to the idea that the standard pattern found in Experiments 1 and 2 is due to the unfamiliarity of the faces. Indeed, this pattern appears to differentiate between familiar and unfamiliar faces.

If the association between target-present and targetabsent performance is really a function of familiarity, with familiar faces giving a pattern consistent with the mirror effect, we would expect the effect to be disrupted by manipulations which disrupt the processing of identity. There is good evidence in the literature that inverted faces are not processed in the same manner as upright faces (e.g., Yin, 1969). Rather, they are processed in a manner more similar to the general object processing system (de Gelder \& Rouw, 2000; Farah, Wilson, Drain, \& Tanaka, 1995; Haxby et al., 1999; Moscovitch, Winocur, \& Behrmann, 1997). 
Table 4

Mean Performance (\%) on Matching Unfamiliar and Familiarized Faces (By-Item Analysis) in Experiment 3B

\begin{tabular}{|c|c|c|c|c|c|c|c|c|c|c|}
\hline & \multicolumn{6}{|c|}{ Target-Present Trials } & \multicolumn{4}{|c|}{ Target-Absent Trials } \\
\hline & \multicolumn{2}{|c|}{ Hits } & \multicolumn{2}{|c|}{ Misses } & \multicolumn{2}{|c|}{ Misidentifications } & \multicolumn{2}{|c|}{ Correct Reject } & \multicolumn{2}{|c|}{ FPs } \\
\hline & $M$ & $S D$ & $M$ & $S D$ & $M$ & $S D$ & $M$ & $S D$ & $M$ & $S D$ \\
\hline Unfamiliar & 69 & 19 & 20 & 14 & 11 & 10 & 70 & 19 & 30 & 19 \\
\hline Familiarized & 87 & 16 & 10 & 12 & 3 & 6 & 88 & 14 & 11 & 14 \\
\hline
\end{tabular}

Note-FPs, false positives.

Therefore, in the final experiment we first familiarized subjects with faces but performed the matching task with inverted faces. This test has been shown to be difficult, but not impossible, by Megreya and Burton (2006b), and so makes a useful technique for understanding the mechanisms underlying the results of Experiments $3 \mathrm{~A}$ and 3B.

\section{EXPERIMENT 4}

\section{Method}

Subjects. Thirty new subjects from the University of Glasgow, 17 women and 13 men, age range 17-27, participated in the experiment for payment or course credits. All reported normal or corrected-tonormal vision.

Stimuli and Procedure. The stimuli and procedure were the same as those in Experiment 3A, with the sole exception that subjects were tested with an inverted face-matching task after the familiarization phase.

\section{Results}

Table 5 shows mean matching performance for inverted faces which had been familiarized. There was no reliable correlation between hits and FPs $[r(38)=-.23, p>.05]$.

\section{Discussion}

In this experiment, subjects were familiarized with faces by the same procedures used in Experiment $3 \mathrm{~A}$, which had been successful in producing the expected negative correlation between hits and FPs. However, once these familiar faces are inverted, the correlation disappears. Since inversion is known to disrupt processes underlying identification, we take this as further evidence that familiarity with the faces is the key factor underlying the existence of an association between target-present and target-absent trials.

\section{GENERAL DISCUSSION}

Our main aim in this article was to examine how familiarity (previous experience) affects the relationship between the ability to identify a target face and to reject a foil using face-matching tasks. A number of interest- ing findings were obtained. First, people are very poor at matching unfamiliar faces, a finding first reported by Bruce et al. (1999), but which is often ignored in theories which conflate familiar and unfamiliar face processing.

Second, there was no relationship between hits and FPs in matching unfamiliar faces in either by-subject or byitem analyses. These findings persisted across very different types of matching task (Experiments 1 and 2). This runs counter to the mirror effect, and possibly counter to intuition (which might suggest that there are some faces which are recognizable and some which are not).

Third, familiarization resulted in a strong negative association between hits and FPs in matching faces. Subjects showed a close to zero correlation between hits and FPs when they were asked to match unfamiliar faces. However, the same subjects showed a very strong negative association when they had been familiarized with the targets before performing the face-matching task. This is most striking, because it suggests that the key characteristic of conditions in which one observes mirror-like effects is that the stimuli are familiar. Further support for this proposal comes from the final experiment, in which familiar faces are rendered "unfamiliar" by inversion, and in which the association disappears.

These findings have significant theoretical implications for the dissociation between the processing of familiar and unfamiliar faces. Early work in this field highlighted differences between the processing of familiar and unfamiliar faces (see Bruce, 1986; Klatzky \& Forrest, 1984; Malone, Morris, Kay, \& Levin, 1982; Young, Hay, McWeeny, Flude, \& Ellis, 1985; Young, Newcombe, de Haan, Small, \& Hay, 1993). Familiar faces are associated with rich semantics, and recognition is not disrupted by minor image variations, whereas unfamiliar faces appear to be treated in a much more superficial, image-dependent fashion (Hancock et al., 2000). The relationship between hits and FPs seems to differentiate between familiar and unfamiliar face processing in a very neat way, and may act as an index of familiarity.

This may also be the key to understanding why the ubiquitous mirror effect is not present in recognition memory

Table 5

Mean Subjects' Performance (\%) in Experiment 4 Target-Present Trials Target-Absent Trials

\begin{tabular}{|c|c|c|c|c|c|c|c|c|c|}
\hline \multicolumn{6}{|c|}{ Target-Present Trials } & \multicolumn{4}{|c|}{ Target-Absent Trials } \\
\hline \multicolumn{2}{|c|}{ Hits } & \multicolumn{2}{|c|}{ Misses } & \multicolumn{2}{|c|}{ Misidentifications } & \multicolumn{2}{|c|}{ Correct Reject } & \multicolumn{2}{|c|}{ FPs } \\
\hline$M$ & $S D$ & $M$ & $S D$ & $M$ & $S D$ & $M$ & $S D$ & $M$ & $S D$ \\
\hline 65 & 16 & 24 & 13 & 11 & 9 & 70 & 23 & 30 & 23 \\
\hline
\end{tabular}


for faces. In fact, all previous tests have used unfamiliar faces, whereas tests of the mirror effect with other stimuli suggest that familiarity may be an important dimension. Studies using meaningless stimuli, such as pseudowords (Maddox \& Estes, 1997; Reder, Angstadt, Cary, Erickson, \& Ayers, 2002) or Chinese characters (Nelson \& Shiffrin, 2005), show an absence of a mirror effect. In contrast, high levels of familiarization were successful in producing the mirror effect for pseudowords (Reder et al., 2002) and Chinese characters (Nelson \& Shiffrin, 2005). These results are compatible with our present findings, where the mirror effect did not appear for faces unless they were familiarized.

Together, the results support single-factor theories (e.g., Glanzer et al., 1993; McClelland \& Chappell, 1998; Shiffrin \& Steyvers, 1997), holding that familiarity is the basis of the mirror effect: People with repeated exposure become better at identifying items as old when old and as new when new. In contrast, dual factor theories (e.g., Joordens \& Hockley, 2000; Reder et al., 2000) give significance to recollection as well as to familiarity. For example, Reder et al. (2002) suggested that the mirror effect is caused by a higher rate of recollection-based hits to low-frequency items than to high-frequency ones, and by a higher rate of familiarity-based FPs to high-frequency items than to low-frequency ones. Nevertheless, because our data rely primarily on encoding rather than on memory, they fit most straightforwardly with the single factor theories suggesting that familiarity breeds differentiation (e.g., McClelland \& Chappell, 1998).

Importantly, however, familiarity did not produce a mirror effect when we presented faces upside down. This finding is particularly interesting, confirming our previous suggestion that inversion makes familiar faces unfamiliar (Megreya \& Burton, 2006b). In a recent study, Criss and Shiffrin (2004) provided a consistent pattern of results. During the encoding phase of a word-recognition memory procedure, their subjects were given one of the following tasks: judgment of concreteness, animacy, frequency or pleasantness, vowel counting, spelling, unusual letters, or no task at all. Of all the foregoing, the typical word-frequency mirror effect appeared only when subjects received no instructions or were asked to attend to any unusual letters or words. Other tasks tended to eliminate the hit superiority of low-frequency words, but the FP component remained the same. As Criss and Shiffrin suggested, these tasks might prevent subjects from paying attention to the most distinctive features in low-frequency words, leading to the elimination of hit superiority, confirming the elevated-attention hypothesis (Malmberg \& Nelson, 2003). Therefore, only when stimuli are sufficiently familiar to allow extraction of distinctive elements (configuration for faces and unusual letters for words), apparently, does the mirror effect appear. On the other hand, when this advantage is prevented (by turning faces upside down or by drawing attention to other word characteristics) the mirror effect disappears.

In sum, these data suggest that processing of upright familiar faces is different from processing of upright unfamiliar faces and inverted familiar faces, and the process- ing of upright unfamiliars and inverted familiars is similar to each other. This is consistent with our previous research (Megreya \& Burton, 2006b), and highlights the fact that models of familiar face recognition (Bruce \& Young, 1986; Burton, Bruce, \& Hancock, 1999; Burton, Jenkins, Hancock, \& White, 2005) have little to say on unfamiliar face processing. Similarly, very little is known about the complexity of subjects' differing strategies when they recognize familiar and unfamiliar faces. For example, though we have argued that unfamiliar faces are processed in more image-like fashion than familiar faces, it remains to be seen how this would affect issues such as criterion. It is possible that when learning a face, the transition from unfamiliar to familiar involves a criterion shift, providing a key to some of the problems raised here. This will be a topic for future research.

In addition to its theoretical importance, the data reported in this article have a very important application to the forensic practice. The results suggest that eyewitnesses' ability to identify a suspect is unrelated to their ability to reject that suspect. It would appear, then, that any search for witnesses who are "good at faces" must incorporate at least two components: being accurate when faces are present, and being accurate when they are absent. This present/absent dichotomy adds to the familiar/ unfamiliar dichotomy in face recognition, and may become as important to understand.

\section{AUTHOR NOTE}

Correspondence concerning this article should be addressed to A. M. Burton, Department of Psychology, University of Glasgow, Glasgow G12 8QQ, Scotland (e-mail: mike@psy.gla.ac.uk).

\section{REFERENCES}

BRUCE, V. (1986). Influences of familiarity on the processing of faces. Perception, 15, 387-397.

Bruce, V., Burton, A. M., \& Dench, N. (1994). What's distinctive about a distinctive face? Quarterly Journal of Experimental Psychology, 47A, 119-141.

Bruce, V., Henderson, Z., Greenwood, K., Hancock, P. J. B., BurTON, A. M., \& Miller, P. (1999). Verification of face identities from images captured on video. Journal of Experimental Psychology: Applied, 5, 339-360.

Bruce, V., Henderson, Z., Newman, C., \& Burton, A. M. (2001). Matching identities of familiar and unfamiliar faces caught on CCTV images. Journal of Experimental Psychology: Applied, 7, 207-218.

Bruce, V., \& Young, A. W. (1986). Understanding face recognition. British Journal of Psychology, 77, 305-327.

Burton, A. M., Bruce, V., \& Hancock, P. J. B. (1999). From pixels to people: A model of familiar face recognition. Cognitive Science, 23, 1-31.

Burton, A. M., Jenkins, R., Hancock, P. J. B., \& White, D. (2005). Robust representations for face recognition: The power of averages. Cognitive Psychology, 51, 256-284.

Burton, A. M., Wilson, S., Cowan, M., \& Bruce, V. (1999). Face recognition in poor-quality video: Evidence from security surveillance. Psychological Science, 10, 243-248.

Clark, S. E., \& Davey, S. L. (2005). The target-to-foils shift in simultaneous and sequential lineups. Law \& Human Behavior, 29, 151-172.

Criss, A. H., \& SHIFFrIN, R. M. (2004). Interactions between study task, study time, and the low-frequency hit rate advantage in recognition memory. Journal of Experimental Psychology: Learning, Memory, \& Cognition, 30, 778-786.

Deffenbacher, K. A., Johanson, J., Vetter, T., \& O’Toole, A. J. 
(2000). The face typicality-recognizability relationship: Encoding or retrieval locus? Memory \& Cognition, 28, 1173-1182.

DE Gelder, B., \& Rouw, R. (2000). Paradoxical configuration effects for faces and objects in prosopagnosia. Neuropsychologia, 38, 1271-1279.

Farah, M. J., Wilson, K. D., Drain, H. M., \& Tanaka, J. R. (1995). The inverted faces inversion effect in prosopagnosia: Evidence for mandatory, face-specific perceptual mechanisms. Vision Research, 35, 2089-2093.

GlanZer, M., \& Adams, J. K. (1985). The mirror effect in recognition memory. Memory \& Cognition, 13, 8-20.

GlanZer, M., \& ADAMS, J. K. (1990). The mirror effect in recognition memory: Data and theory. Journal of Experiment Psychology: Learning, Memory, \& Cognition, 16, 5-16.

Glanzer, M., Adams, J. K., Iverson, G. J., \& Kim, K. (1993). The regularities of recognition memory. Psychological Review, 100, 546-567.

Hancock, P. J. B., Bruce, V., \& Burton, A. M. (2000). Recognition of unfamiliar faces. Trends in Cognitive Sciences, 4, 330-337.

Hancock, P. J. B., Burton, A. M., \& Bruce, V. (1996). Face processing: Human perception and principal components analysis. Memory \& Cognition, 24, 26-40.

Haxby, J. V., Ungerleider, L. G., Clark, V. P., Schouten, J. L., Hoffman, E. A., \& Martin, A. (1999). The effects of face inversion on activity in human neural system for face and object perception. Neuron, 22, 189-199.

Henderson, Z., Bruce, V., \& Burton, A. M. (2001). Matching the faces of robbers captured on video. Applied Cognitive Psychology, $15,445-464$.

Hockley, W. E., Hemsworth, D. H., \& Consoli, A. (1999). Shades of the mirror effect: Recognition of faces with and without sunglasses. Memory \& Cognition, 27, 128-138.

Joordens, S., \& HockLEY, W. E. (2000). Recollection and familiarity through the looking glass: When old does not mirror new. Journal of Experimental Psychology: Learning, Memory, \& Cognition, 26, 1534-1555.

Kemp, R., Towell, N., \& Pike, G. (1997). When seeing should not be believing: Photographs, credit cards and fraud. Applied Cognitive Psychology, 11, 211-222.

Klatzky, R. L., \& Forrest, F. H. (1984). Recognizing familiar and unfamiliar faces. Memory \& Cognition, 12, 60-70.

Lewis, M. B., \& Johnston, R. A. (1997). Familiarity, target set, and false positives in face recognition. European Journal of Cognitive Psychology, 9, 437-459.

Liu, C. H., Seetzen, H., Burton, A. M., \& Chaudhuri, A. (2003). Face recognition is robust with incongruent image resolution: Relationship to security video images. Journal of Experimental Psychology: Applied, 9, 33-41.

MadDox, W. T., \& Estes, W. K. (1997). Direct and indirect stimulusfrequency effects in recognition. Journal of Experimental Psychology: Learning, Memory, \& Cognition, 23, 539-559.

Malmberg, K. J., \& Nelson, T. O. (2003). The word frequency effect for recognition memory and the elevated-attention hypothesis. Memory \& Cognition, 31, 35-43.

Malone, D. R., Morris, H. H., Kay, M. C., \& Levin, H. S. (1982). Prosopagnosia: A double dissociation between the recognition of fa- miliar and unfamiliar faces. Journal of Neurology, Neurosurgery, \& Psychiatry, 45, 820-822.

McClelland, J. L., \& Chappell, M. (1998). Familiarity breeds differentiation: A subjective-likelihood approach to the effects of experience in recognition memory. Psychological Review, 105, 724-760.

Megreya, A. M., \& Burton, A. M. (2006a). Recognising faces seen alone or with others: When two heads are worse than one. Applied Cognitive Psychology, 20, 957-972.

Megreya, A. M., \& Burton, A. M. (2006b). Unfamiliar faces are not faces: Evidence from a matching task. Memory \& Cognition, 34, 865876.

Moscovitch, M., Winocur, G., \& Behrmann, M. (1997). What is special about face recognition? Nineteen experiments on a person with visual object agnosia and dyslexia but normal face recognition. Journal of Cognitive Neuroscience, 9, 555-604.

Nelson, A. N., \& Shiffrin, R. M. (2005, November). The effects of experience on perception and memory. Paper presented at the 46th Annual Meeting of the Psychonomic Society, Toronto.

O’Toole, A. J., Deffenbacher, K. A., Valentin, D., \& Abdi, H. (1994) Structural aspects of face recognition and the other-race effect. Memory \& Cognition, 22, 208-224.

Reder, L. M., Angstadt, P., Cary, M., Erickson, M. A., \& Ayers, M. S. (2002). A reexamination of stimulus-frequency effects in recognition: Two mirrors for low- and high-frequency pseudowords. Journal of Experimental Psychology: Learning, Memory, \& Cognition, 28, 138152

Reder, L. M., Nhouyvanisvong, A., Schunn, C. D., Ayers, M. S., Angstadt, P., \& Hiraki, K. (2000). A mechanistic account of the mirror effect for word frequency: A computational model of remember-know judgments in a continuous recognition paradigm. Journal of Experimental Psychology: Learning, Memory, \& Cognition, 26, 294-320.

Shiffrin, R. M., \& Steyvers, M. (1997). A model for recognition memory: REM-retrieving effectively from memory. Psychonomic Bulletin \& Review, 4, 145-166.

SiKström, S. (2004). The variance reaction time model. Cognitive Psychology, 48, 371-421.

Vokey, J. R., \& Read, J. D. (1992). Familiarity, memorability, and the effect of typicality on the recognition of faces. Memory \& Cognition, 20, 291-302.

WeLLS, G. L. (1993). What do we know about eyewitness identification? American Psychologist, 48, 553-571.

YIN, R. K. (1969). Looking at upside-down faces. Journal of Experimental Psychology, 81, 141-145.

Young, A. W., Hay, D. C., McWeeny, K. H., Flude, B. M., \& Ellis, A. W. (1985). Matching familiar and unfamiliar faces on internal and external features. Perception, 14, 737-746.

Young, A. W., Newcombe, F., de Haan, E. H. F., Small, M., \& Hay, D. C. (1993). Face perception after brain injury: Selective impairments affecting identity and expression. Brain, 116, 941-959.

(Manuscript received August 7, 2006; revision accepted for publication March 26, 2007.) 